\title{
WHITTAKER FUNCTIONS ASSOCIATED TO NEWFORMS FOR GL $(n)$ OVER $p$-ADIC FIELDS
}

\author{
MICHITAKA MIYAUCHI
}

\begin{abstract}
Let $F$ be a non-archimedean local field of characteristic zero. Jacquet, PiatetskiShapiro and Shalika introduced the notion of newforms for irreducible generic representations of $\mathrm{GL}_{n}(F)$. In this paper, we give an explicit formula for Whittaker functions associated to newforms on the diagonal matrices in $\mathrm{GL}_{n}(F)$.
\end{abstract}

\section{INTRODUCTION}

Let $F$ be a non-archimedean local field of characteristic zero. Shintani [12] gave an explicit formula for spherical Whittaker functions of unramified principal series representations of $\mathrm{GL}_{n}(F)$. His formula is a key to the unramified computation of Rankin-Selberg type zeta integrals (see for example [2] and [3]). In this paper, we extend Shintani's result to Whittaker functions associated to newforms for $\mathrm{GL}_{n}(F)$.

Jacquet, Piatetski-Shapiro and Shalika [7] introduced the notion of newforms for irreducible generic representations of $\mathrm{GL}_{n}(F)$, which is an extension of that for $\mathrm{GL}_{2}(F)$ by Casselman [4]. Newforms for $\mathrm{GL}_{n}(F)$ are defined by using a certain family of open compact subgroups $\left\{K_{n}\right\}_{n \geq 0}$. Given an irreducible generic representation $\pi$ of $\mathrm{GL}_{n}(F)$, the smallest integer $c(\pi)$ among those $n$ such that $\pi$ has $K_{n}$-fixed vectors is called the conductor of $\pi$. We say that a vector in $\pi$ is a newform if it is fixed by $K_{c(\pi)}$. When the conductor of $\pi$ is zero, its newforms are just $\mathrm{GL}_{n}(\mathfrak{o})$-fixed vectors, where $\mathfrak{o}$ is the ring of integers in $F$. In this paper, we give an explicit formula for Whittaker functions associated to newforms on the diagonal matrices in $\mathrm{GL}_{n}(F)$.

Originally, Shintani's explicit formula for spherical Whittaker functions is written in terms of Hecke eigenvalues. We will follow his method. For an irreducible generic representation $\pi$, the Hecke algebra associated to $K_{c(\pi)}$ acts on the space of its newforms. Since this space is onedimensional, the actions of elements in this Hecke algebra are given by scalar multiplication. Suppose that the conductor of $\pi$ is positive. Then, similar to the unramified case, we get a formula for the Whittaker function $W$ associated to a newform on $T_{1}=\left\{\operatorname{diag}\left(a_{1}, \ldots, a_{n-1}, 1\right) \mid a_{i} \in F^{\times}\right\}$ in terms of Hecke eigenvalues $\lambda_{1}, \ldots, \lambda_{n-1}$ (see section 3 for precise definition). Kondo and Yasuda [9] showed the relation between these Hecke eigenvalues and the $L$-factor of $\pi$. We therefore obtain an explicit formula for $W$ on $T_{1}$ in terms of the $L$-factor of $\pi$ (Theorem 4.1).

As a corollary, we show that Whittaker functions associated to newforms attain $L$-factors when they are integrated on $\mathrm{GL}_{1}(F)$ which is embedded into the upper left side in $\mathrm{GL}_{n}(F)$ (Theorem 5.1).

We note that our formula determines Whittaker functions associated to newforms for $\pi$ on $B K_{c(\pi)}$, where $B$ denotes the upper triangular Borel subgroup of $\mathrm{GL}_{n}(F)$. But the set $B K_{c(\pi)}$ is smaller than $\mathrm{GL}_{n}(F)$ when the conductor of $\pi$ is positive. However it seems that our formula is enough to compute several kinds of zeta integrals when all data in them are related to newforms.

Recently, Matringe [11] gave a constructive proof of the existence of newforms for generic representations $\pi$ of $\mathrm{GL}_{n}(F)$, by investigating derivatives of $\pi$. One can find the same formula for Whittaker functions associated to newforms in loc. cit.

2010 Mathematics Subject Classification. Primary 22E50, 22E35.

Key words and phrases. local newform, Whittaker function. 
Acknowledgements The author would like to thank Satoshi Kondo, Takuya Yamauchi and Seidai Yasuda for helpful discussions and comments. He also thanks the referee for useful suggestions.

\section{LOCAL NEWFORMS}

In this section, we recall from [7] the notion and basic properties of local newforms for GL $(n)$. Let $F$ be a non-archimedean local field of characteristic zero, $\mathfrak{o}$ its ring of integers, $\mathfrak{p}$ the maximal ideal in $\mathfrak{o}$, and $\varpi$ a generator of $\mathfrak{p}$. We write $|\cdot|$ for the absolute value of $F$ normalized so that $|\varpi|=q^{-1}$, where $q$ denotes the cardinality of the residue field $\mathfrak{o} / \mathfrak{p}$. We fix a non-trivial additive character $\psi$ of $F$ whose conductor is $\mathfrak{o}$.

We set $G=\mathrm{GL}_{n}(F)$. Let $B$ denote the Borel subgroup of $G$ consisting of the upper triangular matrices, and $U$ its unipotent radical. We use the same letter $\psi$ for the following character of $U$ induced from $\psi$ :

$$
\psi(u)=\psi\left(\sum_{i=1}^{n-1} u_{i, i+1}\right), \text { for } u=\left(u_{i, j}\right) \in U .
$$

Let $(\pi, V)$ be an irreducible generic representation of $G$. Then there exists a unique element $l$ in $\operatorname{Hom}_{U}(\pi, \psi)$ up to constant. For $v \in V$, we define the Whittaker function associated to $v$ by

$$
W_{v}(g)=l(\pi(g) v), g \in G .
$$

We call $\mathcal{W}(\pi, \psi)=\left\{W_{v} \mid v \in V\right\}$ the Whittaker model of $\pi$ with respect to $\psi$.

Put $K_{0}=\mathrm{GL}_{n}(\mathfrak{o})$. For each positive integer $m$, let $K_{m}$ be the subgroup of $K_{0}$ consisting of the elements $k=\left(k_{i j}\right)$ in $K_{0}$ which satisfy

$$
\left(k_{n 1}, k_{n 2}, \ldots, k_{n n}\right) \equiv(0,0, \ldots, 0,1) \quad\left(\bmod \mathfrak{p}^{m}\right) .
$$

We write $V(m)$ for the space of $K_{m}$-fixed vectors in $V$. Due to [7] (5.1) Théorème (ii), there exists a non-negative integer $m$ such that $V(m) \neq\{0\}$. We denote by $c(\pi)$ the smallest integer with this property. We call $c(\pi)$ the conductor of $\pi$, and elements in $V(c(\pi))$ newforms for $\pi$. By [7] (5.1) Théorème (ii), we have

$$
\operatorname{dim} V(c(\pi))=1 .
$$

For simplicity, we say that an element $W$ in $\mathcal{W}(\pi, \psi)$ is a newform if $W$ is the Whittaker function associated to a newform for $\pi$. Suppose that $W$ is a non-zero newform in $\mathcal{W}(\pi, \psi)$. Then by the existence of Kirillov model for $\pi$ (see [1] Theorem 5.20), there exists an element $g \in \mathrm{GL}_{n-1}(F)$ such that

$$
W\left(\begin{array}{cc}
g & \\
& 1
\end{array}\right) \neq 0
$$

For any element $f=\left(f_{1}, \ldots, f_{n-1}\right)$ in $\mathbf{Z}^{n-1}$, we set

$$
\varpi^{f}=\operatorname{diag}\left(\varpi^{f_{1}}, \ldots, \varpi^{f_{n-1}}, 1\right) \in G .
$$

By using the Iwasawa decomposition of $\mathrm{GL}_{n-1}(F)$, we see that there exists $f \in \mathbf{Z}^{n-1}$ such that $W\left(\varpi^{f}\right) \neq 0$.

Proposition 2.2. Let $W$ be a newform in $\mathcal{W}(\pi, \psi)$. For $f \in \mathbf{Z}^{n-1}$, we have $W\left(\varpi^{f}\right)=0$ unless $f_{1} \geq \ldots \geq f_{n-1} \geq 0$.

Proof. The proposition follows since $W$ is $U \cap M_{n}(\mathfrak{o})$-invariant. 


\section{HeCKe OPERATORS}

Let $(\pi, V)$ be an irreducible generic representation of $G$ with conductor $c=c(\pi)$. Throughout this section, we suppose that $c$ is positive. For each $g \in G$, we define the Hecke operator $T_{g}$ on $V(c)$ by

$$
T_{g} v=\frac{1}{\operatorname{vol}\left(K_{c}\right)} \int_{K_{c} g K_{c}} \pi(k) v d k=\sum_{k \in K_{c} / K_{c} \cap g K_{c} g^{-1}} \pi(k g) v,
$$

for $v \in V(c)$. By (2.1), there exists a complex number $\lambda_{g}$ such that $T_{g}=\lambda_{g} 1_{V(c)}$. We call $\lambda_{g}$ the Hecke eigenvalue of $T_{g}$.

For $1 \leq i \leq n-1$, we denote by $\lambda_{i}$ the Hecke eigenvalue of $T_{i}=T_{\varpi f^{i}}$, where

$$
f^{i}=(\overbrace{1, \ldots, 1}^{i}, 0, \ldots, 0) \in \mathbf{Z}^{n-1} .
$$

To describe $T_{i}$, we give a complete system of representatives for $K_{m} / K_{m} \cap \varpi^{f^{i}} K_{m} \varpi^{-f^{i}}(m>0$, $1 \leq i \leq n-1)$. We write $A \in M_{n}(F)$ as

$$
A=\left(\begin{array}{cc}
A_{11} & A_{12} \\
A_{21} & A_{22}
\end{array}\right)
$$

where $A_{11} \in M_{n-1}(F), A_{12} \in M_{n-1,1}(F), A_{21} \in M_{1, n-1}(F)$ and $A_{22} \in F$. We embed the group $\mathrm{GL}_{n-1}(F)$ into $G$ by

$$
g \mapsto\left(\begin{array}{cc}
g & \\
& 1
\end{array}\right), g \in \mathrm{GL}_{n-1}(F) .
$$

Then we may regard $\varpi^{f}, f \in \mathbf{Z}^{n-1}$ as an element in $\mathrm{GL}_{n-1}(F)$. Set $H=\mathrm{GL}_{n-1}(\mathfrak{o})$. For $0 \leq i \leq n-1$, we define an $\mathfrak{o}$-lattice $L_{i}$ in $M_{n-1,1}(F)$ by

$$
L_{i}={ }^{t}(\overbrace{\mathfrak{p} \oplus \cdots \oplus \mathfrak{p}}^{i} \oplus \mathfrak{o} \oplus \cdots \oplus \mathfrak{o}) .
$$

Then the groups $H$ and $H \cap \varpi^{f^{i}} H \varpi^{-f^{i}}$ fix $L_{0}$ and $L_{i}$ respectively.

Lemma 3.1. Let $m$ be a positive integer. For $1 \leq i \leq n-1$, we can take

$$
\left(\begin{array}{cc}
a & x \\
0 & 1
\end{array}\right), a \in H / H \cap \varpi^{f^{i}} H \varpi^{-f^{i}}, x \in L_{0} / a L_{i}
$$

as a complete system of representatives for $K_{m} / K_{m} \cap \varpi^{f^{i}} K_{m} \varpi^{-f^{i}}$.

Proof. We use the block notation. An element $g=\left(\begin{array}{ll}a & b \\ c & d\end{array}\right) \in K_{m}$ lies in $K_{m} \cap \varpi^{f^{i}} K_{m} \varpi^{-f^{i}}$ if and only if $a \in H \cap \varpi^{f^{i}} H \varpi^{-f^{i}}$ and $b \in L_{i}$. Thus, one can observe that the elements in the lemma belong to pairwise disjoint cosets in $K_{m} / K_{m} \cap \varpi^{f^{i}} K_{m} \varpi^{-f^{i}}$. For $g=\left(\begin{array}{ll}a & b \\ c & d\end{array}\right) \in K_{m}$, we see that $g$ is equivalent to $\left(\begin{array}{cc}a & b \\ 0 & 1\end{array}\right)$ modulo $K_{m} \cap \varpi^{f^{i}} K_{m} \varpi^{-f^{i}}$. This completes the proof of the lemma.

Let $W$ be a newform in $\mathcal{W}(\pi, \psi)$. Set $w(f)=W\left(\varpi^{f}\right)$, for $f \in \mathbf{Z}^{n-1}$. Then we obtain the following lemma:

Lemma 3.2. Suppose that $f \in \mathbf{Z}^{n-1}$ satisfies $f_{1} \geq \ldots \geq f_{n-1} \geq 0$. Then we have

$$
q^{-i} \lambda_{i} w(f)=q^{i(n-1)-i(i-1) / 2} \sum_{\varepsilon \in I_{i}} q^{-\sum_{j=1}^{n-1} \varepsilon_{j} j} w(f+\varepsilon),
$$

where $I_{i}=\left\{\varepsilon \in \mathbf{Z}^{n-1} \mid \varepsilon_{j} \in\{0,1\}, \sum_{j=1}^{n-1} \varepsilon_{j}=i\right\}$. 
Proof. By Lemma 3.1, we get

$$
\begin{aligned}
\lambda_{i} W\left(\varpi^{f}\right) & =\sum_{k \in K_{c} / K_{c} \cap \varpi^{f^{i}} K_{c} \varpi^{-f^{i}}} W\left(\varpi^{f} k \varpi^{f^{i}}\right) \\
& =\sum_{a \in H / H \cap \varpi^{f^{i} H \varpi^{-f^{i}},}, x \in L_{0} / a L_{i}} W\left(\varpi^{f}\left(\begin{array}{cc}
1 & x \\
0 & 1
\end{array}\right)\left(\begin{array}{cc}
a & 0 \\
0 & 1
\end{array}\right) \varpi^{f^{i}}\right) \\
& =q^{i} \sum_{a \in H / H \cap \varpi^{f^{i} H \varpi^{-f^{i}}}} W\left(\varpi^{f}\left(\begin{array}{cc}
a & 0 \\
0 & 1
\end{array}\right) \varpi^{f^{i}}\right) .
\end{aligned}
$$

In the last equality, we use the equation $\left[L_{0}: a L_{i}\right]=\left[a L_{0}: a L_{i}\right]=\left[L_{0}: L_{i}\right]=q^{i}$. Now the proof is quite similar to that of the theorem in [12] p. 181 because $\left.W\right|_{\mathrm{GL}_{n-1}(F)}$ is $\mathrm{GL}_{n-1}(\mathfrak{o})$-invariant. We note that in [12] one should take the set $I_{i}$ as $I_{i}=\left\{\varepsilon \in \mathbf{Z}^{n} \mid \varepsilon_{j} \in\{0,1\}, \sum_{j=1}^{n} \varepsilon_{j}=i\right\}$.

\section{An EXPlicit FORMUla FOR WhitTAKer FUnCTIONS}

We prepare some notation to state our main theorem. For an irreducible generic representation $\pi$ of $G$, let $L(s, \pi)$ denote its $L$-factor defined in [5]. It follows from [6] section 3 that the degree of $L(s, \pi)$ is equal to or less than $n$. So we can write it as

$$
L(s, \pi)=\prod_{i=1}^{n}\left(1-\alpha_{i} q^{-s}\right)^{-1}, \alpha_{i} \in \mathbf{C} .
$$

By [6] section 3 again, $L(s, \pi)$ is of degree $n$ if and only if $\pi$ is unramified, that is, $c(\pi)$ equals to zero. We take $\alpha_{n}$ to be zero if the degree of $L(s, \pi)$ is less than $n$. Let $X=\left(X_{1}, \ldots, X_{n}\right)$ be $n$ indeterminates. If $f \in \mathbf{Z}^{n}$ satisfies $f_{1} \geq \ldots \geq f_{n} \geq 0$, then we denote by $s_{f}(X)$ the Schur polynomial in $X_{1}, \ldots, X_{n}$ associated to $f$, that is,

$$
s_{f}(X)=\frac{\left|\left(X_{j}^{f_{i}+n-i}\right)_{1 \leq i, j \leq n}\right|}{\prod_{1 \leq i<j \leq n}\left(X_{i}-X_{j}\right)}
$$

(see [10] Chapter I, section 3). Since $s_{f}(X)$ is a symmetric polynomial in $X_{1}, \ldots, X_{n}$, the number $s_{f}(\alpha)=s_{f}\left(\alpha_{1}, \ldots, \alpha_{n}\right)$ is well-defined. We identify $\left(f_{1}, \ldots, f_{n-1}\right) \in \mathbf{Z}^{n-1}$ with $\left(f_{1}, \ldots, f_{n-1}, 0\right) \in$ $\mathbf{Z}^{n}$. We note that if the conductor of $\pi$ is positive, then we have $s_{f}(\alpha)=s_{\left(f_{1}, \ldots, f_{n-1}, 0\right)}\left(\alpha_{1}, \ldots, \alpha_{n-1}, 0\right)=$ $s_{\left(f_{1}, \ldots, f_{n-1}\right)}\left(\alpha_{1}, \ldots, \alpha_{n-1}\right)$, for $f \in \mathbf{Z}^{n-1}$ such that $f_{1} \geq \ldots \geq f_{n-1} \geq 0$.

We denote by $\delta_{B}$ the modulus character of $B$. We have $\delta_{B}\left(\varpi^{f}\right)=q^{-\sum_{j=1}^{n-1}(n+1-2 j) f_{j}}$, for $f \in \mathbf{Z}^{n-1}$.

Theorem 4.1. Let $\pi$ be an irreducible generic representation of $G$ and $W$ its newform in $\mathcal{W}(\pi, \psi)$. For $f \in \mathbf{Z}^{n-1}$, we have

$$
W\left(\varpi^{f}\right)=\left\{\begin{array}{cl}
\delta_{B}^{1 / 2}\left(\varpi^{f}\right) s_{f}(\alpha) W(1), & \text { if } f_{1} \geq \ldots \geq f_{n-1} \geq 0 ; \\
0, & \text { otherwise. }
\end{array}\right.
$$

Proof. If $c(\pi)=0$, then the theorem follows from [12. So we may assume that $\pi$ has positive conductor. For $f \in \mathbf{Z}^{n-1}$, we set

$$
\widetilde{w}(f)=q^{\sum_{j=1}^{n-1}(n-1-j) f_{j}} W\left(\varpi^{f}\right) .
$$

By Proposition 2.2 and Lemma 3.2, the function $\widetilde{w}$ on $\mathbf{Z}^{n-1}$ satisfies the following system of difference equations:

$$
\left\{\begin{array}{cl}
q^{i(i-1) / 2-i} \lambda_{i} \widetilde{w}(f)=\sum_{\varepsilon \in I_{i}} \widetilde{w}(f+\varepsilon), & \text { if } f_{1} \geq \ldots \geq f_{n-1} \geq 0 \\
\widetilde{w}(f)=0, & \text { otherwise. }
\end{array}\right.
$$


As in [12] p. 182, the solution of the above difference equations is unique and given by

$$
\widetilde{w}(f)=\left\{\begin{array}{cl}
s_{f}(\mu) W(1), & \text { if } f_{1} \geq \ldots \geq f_{n-1} \geq 0 \\
0, & \text { otherwise }
\end{array}\right.
$$

where $\mu_{1}, \ldots, \mu_{n-1}$ are complex numbers whose $i$-th elementary symmetric polynomial equals to $q^{i(i-1) / 2-i} \lambda_{i}$, for $1 \leq i \leq n-1$, and $\mu_{n}=0$.

By [9] Theorem 4.2, we have

$$
L(s, \pi)=\left(\sum_{i=0}^{n-1}(-1)^{i} \lambda_{i} q^{\frac{i(i-1)}{2}-i\left(\frac{n-1}{2}+s\right)}\right)^{-1} .
$$

Hence we may assume $\mu_{i}=q^{(n-1) / 2-1} \alpha_{i}$, for $1 \leq i \leq n$. Thus, if $f \in \mathbf{Z}^{n-1}$ satisfies $f_{1} \geq \ldots \geq$ $f_{n-1} \geq 0$, then we obtain

$$
\begin{aligned}
W\left(\varpi^{f}\right) & =q^{-\sum_{j=1}^{n-1}(n-1-j) f_{j}} s_{f}(\mu) W(1)=q^{-\sum_{j=1}^{n-1}(n-1-j) f_{j}+\left(\frac{n-1}{2}-1\right) \sum_{j=1}^{n-1} f_{j}} s_{f}(\alpha) W(1) \\
& =q^{-\sum_{j=1}^{n-1}\left(\frac{n+1}{2}-j\right) f_{j}} s_{f}(\alpha) W(1)=\delta_{B}^{1 / 2}\left(\varpi^{f}\right) s_{f}(\alpha) W(1) .
\end{aligned}
$$

This completes the proof.

Remark 4.3. Set $D_{1}=\left\{\varpi^{f} \mid f \in \mathbf{Z}^{n-1}\right\}$. Since the center $Z$ of $G$ acts on $\mathcal{W}(\pi, \psi)$ by the central character of $\pi$, Theorem 4.1 gives an explicit formula for newforms in $\mathcal{W}(\pi, \psi)$ on $B K_{c(\pi)}=$ $U Z D_{1} K_{c(\pi)}$.

Corollary 4.4. Let $\pi$ be an irreducible generic representation of $G$. Then we have $W(1) \neq 0$ for all non-zero newforms $W$ in $\mathcal{W}(\pi, \psi)$.

Proof. By Theorem 4.1, $W(1)=0$ implies $W\left(\varpi^{f}\right)=0$ for all $f \in \mathbf{Z}^{n-1}$. This contradicts the remark before Proposition 2.2.

\section{AN APPLICATION TO ZETA INTEGRAL}

In this section, we give an integral representation of $L$-factors by using our formula for Whittaker functions associated to newforms. Let $(\pi, V)$ be an irreducible generic representation of $G$. For $W \in \mathcal{W}(\pi, \psi)$, we set

$$
Z(s, W)=\int_{F^{\times}} W(t(a))|a|^{s-\frac{n-1}{2}} d^{\times} a, s \in \mathbf{C},
$$

where $t(a)=\operatorname{diag}(a, 1, \ldots, 1)$, for $a \in F^{\times}$. Here we normalize Haar measure $d^{\times} a$ on $F^{\times}$so that $\int_{\mathfrak{o}^{\times}} d^{\times} a=1$. The integral $Z(s, W)$ absolutely converges to a rational function in $q^{-s}$ when the real part of $s$ is sufficiently large. By [8] Theorem 2.7 (ii), the set $\{Z(s, W) \mid W \in \mathcal{W}(\pi, \psi)\}$ coincides with the fractional ideal of $\mathbf{C}\left[q^{-s}, q^{s}\right]$ generated by $L(s, \pi)$. We shall show that $Z(s, W)$ attains $L(s, \pi)$ when $W$ is a newform.

Theorem 5.1. Let $\pi$ be an irreducible generic representation of $G$ and $W$ the newform in $\mathcal{W}(\pi, \psi)$ such that $W(1)=1$. Then we have

$$
Z(s, W)=L(s, \pi) .
$$

Proof. By Proposition 2.2 and Theorem 4.1, we obtain

$$
\begin{aligned}
Z(s, W) & =\sum_{k=0}^{\infty} W\left(t\left(\varpi^{k}\right)\right)\left|\varpi^{k}\right|^{s-\frac{n-1}{2}}=\sum_{k=0}^{\infty} \delta_{B}^{1 / 2}\left(t\left(\varpi^{k}\right)\right) s_{(k, 0, \ldots, 0)}(\alpha)\left|\varpi^{k}\right|^{s-\frac{n-1}{2}} \\
& =\sum_{k=0}^{\infty} s_{(k, 0, \ldots, 0)}(\alpha) q^{-k s} .
\end{aligned}
$$


It follows from [10] Chapter I (3.4) that $s_{(k, 0, \ldots, 0)}(\alpha)$ is the complete homogeneous symmetric polynomial of degree $k$, that is,

$$
s_{(k, 0, \ldots, 0)}(\alpha)=\sum_{k_{1}+\cdots+k_{n}=k} \alpha_{1}^{k_{1}} \cdots \alpha_{n}^{k_{n}} .
$$

Hence we get

$$
\begin{aligned}
Z(s, W) & =\sum_{k=0}^{\infty}\left(\sum_{k_{1}+\cdots+k_{n}=k} \alpha_{1}^{k_{1}} \cdots \alpha_{n}^{k_{n}}\right) q^{-k s}=\prod_{i=1}^{n}\left(\sum_{k_{i}=0}^{\infty} \alpha_{i}^{k_{i}} q^{-k_{i} s}\right) \\
& =\prod_{i=1}^{n}\left(1-\alpha_{i} q^{-s}\right)^{-1}=L(s, \pi),
\end{aligned}
$$

as required.

\section{REFERENCES}

[1] I. N. Bernšteĭn and A. V. Zelevinskiı̌. Representations of the group $G L(n, F)$, where $F$ is a local nonArchimedean field. Uspehi Mat. Nauk, 31(3(189)):5-70, 1976.

[2] D. Bump and S. Friedberg. The exterior square automorphic L-functions on GL(n). In Festschrift in honor of I. I. Piatetski-Shapiro on the occasion of his sixtieth birthday, Part II (Ramat Aviv, 1989), volume 3 of Israel Math. Conf. Proc., pages 47-65. Weizmann, Jerusalem, 1990.

[3] D. Bump and D. Ginzburg. Symmetric square L-functions on GL(r). Ann. of Math. (2), 136(1):137-205, 1992.

[4] W. Casselman. On some results of Atkin and Lehner. Math. Ann., 201:301-314, 1973.

[5] R. Godement and H. Jacquet. Zeta functions of simple algebras. Lecture Notes in Mathematics, Vol. 260. Springer-Verlag, Berlin, 1972.

[6] H. Jacquet. Principal $L$-functions of the linear group. In Automorphic forms, representations and L-functions (Proc. Sympos. Pure Math., Oregon State Univ., Corvallis, Ore., 1977), Part 2, Proc. Sympos. Pure Math., XXXIII, pages 63-86. Amer. Math. Soc., Providence, R.I., 1979.

[7] H. Jacquet, I. Piatetski-Shapiro, and J. Shalika. Conducteur des représentations du groupe linéaire. Math. Ann., 256(2):199-214, 1981.

[8] H. Jacquet, I. I. Piatetskii-Shapiro, and J. A. Shalika. Rankin-Selberg convolutions. Amer. J. Math., 105(2):367-464, 1983.

[9] S. Kondo and S. Yasuda. Local L and epsilon factors in Hecke eigenvalues, preprint, IPMU preprints, IPMU100107, 2010. available at http://db.ipmu.jp/ipmu/ipmuno/ipmu_list/.

[10] I. G. Macdonald. Symmetric functions and Hall polynomials. Oxford Mathematical Monographs. The Clarendon Press Oxford University Press, New York, second edition, 1995. With contributions by A. Zelevinsky, Oxford Science Publications.

[11] N. Matringe. Essential Whittaker functions for GL(n) over a $p$-adic field, preprint, arXiv:1201.5506, 2012.

[12] T. Shintani. On an explicit formula for class-1 "Whittaker functions" on $G L_{n}$ over $\mathfrak{P}$-adic fields. Proc. Japan Acad., 52(4):180-182, 1976.

Department of Mathematics, Faculty of Science, Kyoto University, Oiwake Kita-Shirakawa SAKYO KYOTO 606-8502 JAPAN

E-mail address: miyauchi@math.kyoto-u.ac.jp 